\title{
Realization of a Universal Quantum Gate Set for Itinerant Microwave Photons
}

\author{
Kevin Reuer®, ${ }^{1,}$ Jean-Claude Besse $\odot,{ }^{1}$ Lucien Wernli, ${ }^{1}$ Paul Magnard $\odot,{ }^{1}$ Philipp Kurpiers, ${ }^{1}$ \\ Graham J. Norris, ${ }^{1}$ Andreas Wallraff ${ }^{1,2}$ and Christopher Eichler ${ }^{1,}$ \\ ${ }^{1}$ Department of Physics, ETH Zurich, CH-8093 Zurich, Switzerland \\ ${ }^{2}$ Quantum Center, ETH Zurich, CH-8093 Zurich, Switzerland
}

(Received 15 June 2021; accepted 10 November 2021; published 12 January 2022; corrected 16 February 2022)

\begin{abstract}
Deterministic photon-photon gates enable the controlled generation of entanglement between mobile carriers of quantum information. Such gates have thus far been exclusively realized in the optical domain and by relying on postselection. Here, we present a nonpostselected, deterministic, photon-photon gate in the microwave frequency range realized using superconducting circuits. We emit photonic qubits from a source chip and route those qubits to a gate chip with which we realize a universal gate set by combining controlled absorption and reemission with single-qubit gates and qubit-photon controlled-phase gates. We measure quantum process fidelities of $75 \%$ for single- and of $57 \%$ for two-qubit gates, limited mainly by radiation loss and decoherence. This universal gate set has a wide range of potential applications in superconducting quantum networks.
\end{abstract}

DOI: 10.1103/PhysRevX.12.011008

Photons are ideal carriers of quantum information, because they do not interact with each other when propagating through linear media. Such interactions, on the other hand, are essential for the implementation of photonphoton gates [1], which can be used within quantum networks $[2,3]$ or in distributed quantum computing [3-6]. Photon-photon gates enable, for example, the processing of quantum information while photonic qubits are traveling between local quantum processing units, potentially allowing for simpler network structures [7]. All-photonic quantum computing paradigms, such as linear optics [1,7-9], continuous-variable [10,11], and photonic one-way [12-14] quantum computing also rely on photonphoton gates.

Probabilistic photon-photon gates are based on beamsplitter-induced interference effects and projective measurements [1,8]. In particular, the bosonic symmetry relation [1] gives rise to the Hong-Ou-Mandel effect [15], providing a resource for entanglement generation. Together with the nonlinearity of photon detection, interference effects enable (probabilistic) Bell measurements $[1,16]$ as used in quantum repeater protocols [17-20]. By additionally using ancilla measurements and classical

\footnotetext{
*kevin.reuer@phys.ethz.ch

†eichlerc@phys.ethz.ch
}

Published by the American Physical Society under the terms of the Creative Commons Attribution 4.0 International license. Further distribution of this work must maintain attribution to the author(s) and the published article's title, journal citation, and DOI.
Subject Areas: Condensed Matter Physics

Quantum Physics, Quantum Information feed forward, probabilistic photon-photon gates have been implemented in optical setups [21-26] and are now commonly used in linear optics quantum computing [26-28].

Alternatively, interactions of photons with nonlinear media enable deterministic photon-photon gates [29]. Such gates have recently been implemented in the optical domain, either based on photons sequentially reflecting off of an atom in a cavity [30] or based on the transmission through an ensemble of Rydberg atoms after absorption of a control photon [31]. Albeit both implementations [30,31] are deterministic, they use postselection to compensate for losses in the experimental setup.

Here, we exploit the controllable coupling between superconducting qubits and itinerant microwave fields to implement a deterministic, nonpostselected, photon-photon controlled-phase (CPHASE) gate. To this aim, we absorb a first photonic qubit using a stationary gate qubit, reflect off a second photonic qubit of the gate qubit to perform the entangling gate, and reemit the first one. Additionally, we demonstrate universal single-qubit gates acting on the photonic qubits. Since we encode photonic qubits in the single-rail basis as single-photon microwave fields, single-qubit gates do not preserve the photon number and can thus not be realized with linear optics elements [1].

To generate photonic qubits and to temporarily store and act on qubit states, we use a source qubit $S$ and a gate qubit $G$, respectively. Both the source qubit $S$ and the gate qubit $G$ are tunably coupled to the microwave field in a waveguide; see the schematic in Fig. 1. We first generate a train 


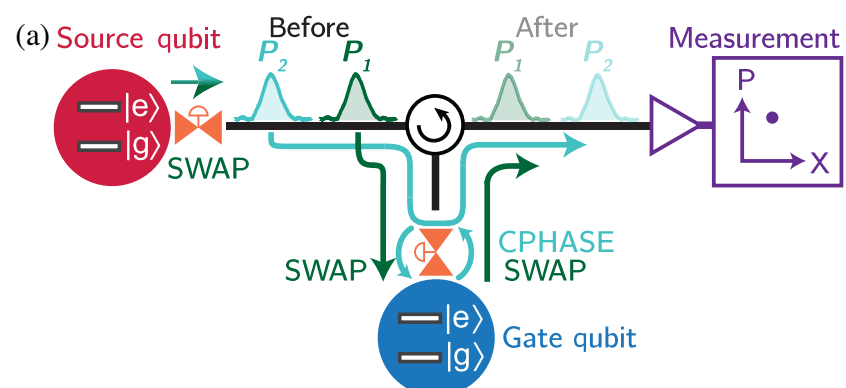

(b)

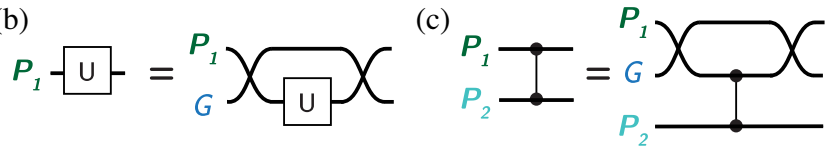

FIG. 1. (a) Schematic representation of the concept of the experiment. We generate photonic qubits $P_{1}$ (dark green) and $P_{2}$ (turquoise) from the source qubit (red) via swap gates (SWAP) mediated by a tunable coupling depicted as a control valve (orange). The photonic qubits propagate through a circulator to the gate qubit (blue), performing a swap gate (SWAP) or a controlled-phase gate (CPHASE). We linearly amplify the output field and measure the photonic qubits using heterodyne detection (purple). (b),(c) Equivalent quantum circuits implemented to realize (b) an arbitrary single-qubit gate $U$ and (c) a photonphoton controlled-phase (CPHASE) gate.

of itinerant photonic qubits $P_{i}$ in the single-rail basis by performing swap gates between the source qubit $S$ and the itinerant field carrying the photonic qubits $P_{i}$ [32-34], as shown in Fig. 1(a). The photonic qubits $P_{i}$ propagate through a circulator toward the gate qubit $G$ [Fig. 1(a)], which implements both single- and two-qubit gates. To realize single-qubit gates acting on $P_{1}$, we first absorb $P_{1}$ by the gate qubit $G$ using the same swap gate as used for photon emission. We then apply a single-qubit unitary $U$ on the gate qubit $G$ and finally perform a second swap to reemit the photonic qubit $P_{1}$; see the equivalent quantum circuit in Fig. 1(b). For the controlled-phase gate [Fig. 1(c)] acting on two photonic qubits $P_{1}$ and $P_{2}$, we first absorb the control qubit $P_{1}$ and then reflect the target qubit $P_{2}$ off of the gate qubit $G$, resulting in a controlled-phase gate between $P_{2}$ and $G$ [35,36]. Finally, we reemit the control qubit $P_{1}$. The control qubit $P_{1}$ is thus shifted from its original time bin to a later time bin, while the target qubit $P_{2}$ remains in its original time bin. After interacting with the gate qubit $G$, the photonic qubits $P_{i}$ propagate through the circulator to the output port at which we measure the field quadratures $X$ and $P$ of the photonic qubits $P_{i}$ using linear amplification and heterodyne detection to tomographically reconstruct quantum states and processes $[32,37]$. Importantly, by realizing the controlled-phase gate between a stationary qubit and an itinerant photonic qubit, we avoid the need for absorbing both photonic qubits into separate stationary modes on the same chip and performing a two-qubit gate between those stationary modes. This choice substantially reduces the resource requirements and avoids the additional time delays encountered during absorption and emission. To perform multiqubit unitaries on several photonic qubits, the described scheme could also be used in combination with a multimode quantum memory on the gate chip, allowing one to sequentially apply several single- and two-qubit gates on different photonic qubits, while storing noninteracting qubits in the memory.

We experimentally realize the described scheme for single- and two-photon gates by using two copies of the superconducting chip presented in Ref. [32] (optical micrograph in the Appendix A)—one for the source qubit $S$ and one for the gate qubit $G$; see Fig. 2(a) for an equivalent electrical circuit diagram. The source qubit $S$ (gate qubit $G$ ) is realized as a flux-tunable transmon qubit tuned to its maximum transition frequency at $\omega_{S, g e} / 2 \pi=5.925 \mathrm{GHz}$ $\left(\omega_{G, g e} / 2 \pi=5.771 \mathrm{GHz}\right)$, coupled to the transmission line via a tunable coupler at $\omega_{S, c} / 2 \pi=3.2 \mathrm{GHz}$ $\left(\omega_{G, c} / 2 \pi=4.6 \mathrm{GHz}\right)$ and a converter mode realized as a transmon with an anharmonicity $\alpha / 2 \pi=260 \mathrm{MHz}$, biased to $\omega_{01} / 2 \pi=5.998 \mathrm{GHz}$. We perform local unitaries by applying microwave pulses via dedicated charge lines. For performing the swap gate, we activate the interaction Hamiltonian $\quad H_{\text {SWAP }}=\hbar J(|e 0\rangle\langle g 1|+| g 1\rangle\langle e 0|)$, where $|g\rangle$ and $|e\rangle$ denote the ground and first excited state of the source qubit (gate qubit), respectively, and $|0\rangle$ and $|1\rangle$ denote the ground and first excited state of the converter mode, respectively, which couples with constant rate $\kappa_{S} / 2 \pi=1.8 \mathrm{MHz}\left(\kappa_{G} / 2 \pi=2.1 \mathrm{MHz}\right)$ to a transmission line into which the propagating fields are emitted. Thus, the converter mode frequency sets the carrier frequency $\omega_{01} / 2 \pi=5.998 \mathrm{GHz}$ of the generated photonic mode. As shown in Fig. 2(b), we realize the interaction Hamiltonian $H_{\text {SWAP }}$ by parametrically driving the transition $|e 0\rangle \leftrightarrow|g 1\rangle$ via a magnetic flux pulse $\Phi(t)$ applied to the superconducting quantum interference device (SQUID) loop of the coupler with a carrier frequency matching the level detuning $\Delta_{S}=\omega_{01} / 2 \pi-\omega_{S, g e} / 2 \pi=$ $73 \mathrm{MHz}\left(\Delta_{G}=\omega_{01} / 2 \pi-\omega_{G, g e} / 2 \pi=227 \mathrm{MHz}\right)$. To optimize the absorption efficiency, we shape the temporal profile of the photonic mode $\xi(t) \propto \operatorname{sech}(\Gamma t / 2)$ to be timesymmetric with bandwidth $\Gamma$ [38-40], by controlling the interaction strength $J$ (see Appendix B for details). We further optimize the absorption process by tuning the converter modes of the source and gate device into resonance with each other (see Appendix $\mathrm{C}$ for details).

To demonstrate our ability to emit and absorb photonic modes with a time-symmetric profile, we measure the average field amplitude vs time of photonic qubits prepared in the state $|+\rangle=(|0\rangle+|1\rangle) / \sqrt{2}$ in three different experimental settings [Fig. 2(c)]. First, we verify the emission of shaped photonic modes by emitting a $|+\rangle$ state from the source qubit $S$ with the converter mode of the gate chip detuned, to ensure the photonic mode reflects off of it without encountering any mode distortions. The measured average field amplitude (red dots) is in good agreement 

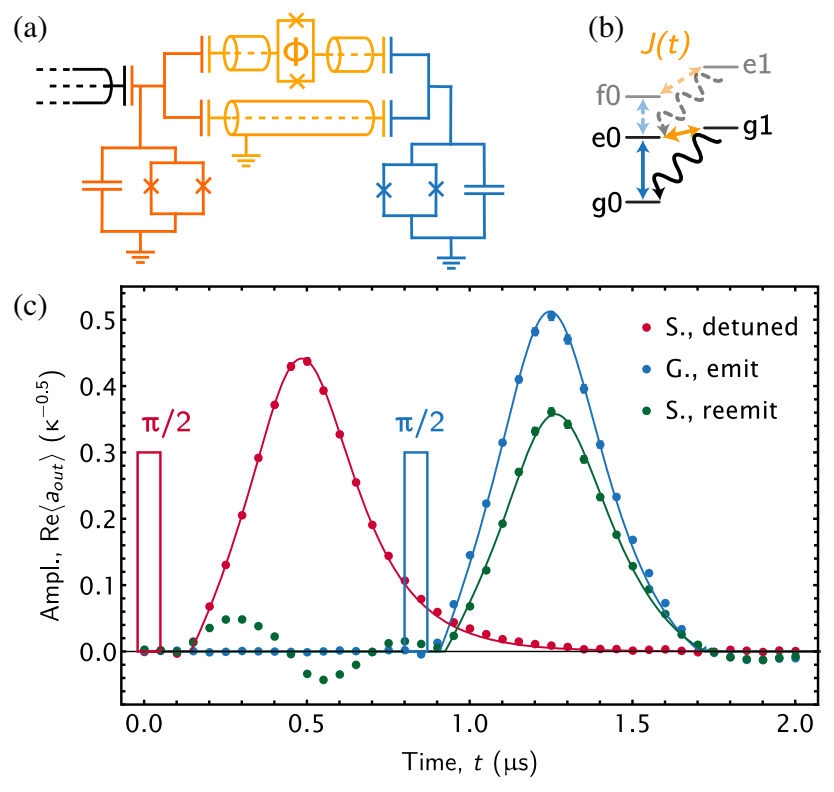

FIG. 2. (a) Electrical circuit diagram of the gate qubit (blue) interacting with fields in the transmission line (black) through a tunable coupler (orange) and a converter mode (dark orange, realized as a transmon qubit). (b) Energy-level diagram indicating transitions induced by single-qubit gates (blue), parametric modulation of the flux $\Phi$ threading the SQUID loop embedded in the coupler (orange), and photon emission via spontaneous decay (black). (c) Measured (dots) and simulated (solid lines) real part of the envelope of the field amplitude $\operatorname{Re}\left\langle a_{\text {out }}\right\rangle$ vs time $t$ for superposition states $(|0\rangle+|1\rangle) / \sqrt{2}$ emitted from the source qubit with the converter mode of the gate device being detuned from the field (red), when absorbing and reemitting the field at the gate qubit (green), and when directly preparing and emitting the state at the gate qubit (blue). A red (blue) wire frame shows the timing of qubit pulses with a rotation angle of $\pi / 2$ applied to the source (gate) qubit prior to emission. Imaginary parts (not shown) are below $0.07 / \sqrt{\kappa}$ in magnitude.

with the temporal profile extracted from a two-level Rabi model using the calibrated parameters (red line; see Appendix B for details). Second, we demonstrate that we can absorb and reemit shaped photonic modes by emitting a photonic qubit in the $|+\rangle$ state from the source qubit and absorbing and reemitting the photonic qubit at the gate qubit. The measured amplitude (green dots) is also in good agreement with the simulated one (green line) but is $19 \%$ smaller in amplitude [compared to the photonic qubits emitted from the source qubit with the gate qubit converter mode detuned (red)], mainly due to the additional decoherence encountered during the absorption and reemission by the gate qubit (inferred from a master equation simulation; see Appendix D for details). For comparison and to quantify the photon loss during propagation from the source to the gate device and during the absorption and reemission process, we also emit $|+\rangle$ states directly prepared at the gate qubit (blue dots). We find a ratio between the measured amplitudes of photonic qubits emitted from the source (red, with gate qubit converter mode detuned) and gate qubit (blue) of about $86 \%$, which is close to the transmission efficiency $\sqrt{\eta_{\text {loss }}}=\sqrt{1-0.25}=87 \%$, determined in an independent calibration measurement (see Appendix A for details) and is mainly due to the loss in the circulator. From the measured photon field amplitudes, we estimate that, when we absorb the photonic qubit at the gate qubit, $1-\eta_{\mathrm{abs}}=3 \%$ of the incoming photon flux still reflects off of the gate chip. We attribute the finite absorption efficiency $\eta_{\text {abs }}$ mainly to the truncation of the flux pulse generating the photonic mode $\xi(t) \propto \operatorname{sech}(\Gamma t / 2)$ at times $\pm 4.6 / \kappa_{S}$ (see Appendix B for details).

After having confirmed our ability to absorb and reemit photons, we demonstrate the second ingredient of the photon-photon gate scheme, shown in Fig. 1(c): a controlled-phase gate between the gate qubit $G$ and a photonic mode $P_{i}$. For this purpose, we parametrically tune the two energy levels $|f 0\rangle$ and $|e 1\rangle$, where $|f\rangle$ denotes the second excited state of the gate qubit, into resonance by driving the coupler with an amplitude $A$ at a frequency of $\Delta_{\mathrm{CPHASE}}=\Delta_{G}+\alpha_{G} / 2 \pi=529 \mathrm{MHz}$. Here, $\alpha_{G} / 2 \pi=$ $302 \mathrm{MHz}$ is the anharmonicity of the gate qubit. Transitions from $|e 0\rangle$ into the dressed states $\left|\psi_{+}\right\rangle=(|f 0\rangle+$ $|e 1\rangle) / \sqrt{2}$ and $\left|\psi_{-}\right\rangle=(|f 0\rangle-|e 1\rangle) / \sqrt{2}$ are detuned from the bare converter mode by $g / 2 \pi=1.6 \mathrm{MHz}$, where $g \propto A$ is the parametric interaction strength, which is to first order proportional to the drive amplitude $A$. As in Ref. [35], we expect an incoming photonic mode in resonance with the converter mode to be reflected off of the gate qubit with a $\pi$ phase shift when the gate qubit $G$ is in the ground state $|g\rangle$ while being reflected with no phase shift when the gate qubit $G$ is in the excited state $|e\rangle$ due to the symmetric detuning between the photonic mode and the $|e 0\rangle \leftrightarrow\left|\psi_{+}\right\rangle$ and the $|e 0\rangle \leftrightarrow\left|\psi_{-}\right\rangle$transitions; see the insets in Fig. 3(a). We experimentally observe the predicted $\pi$ phase shift as a sign change in the real part of the average field amplitude [Fig. 3(a)], when preparing the gate qubit in the ground state $|g\rangle$ or in the excited state $|e\rangle$ and emitting a photonic state $|+\rangle$ from the source qubit while driving the coupler of the gate qubit at the frequency $\Delta_{\mathrm{CPHASE}}$. The measured photon field amplitudes (dots) match well with the simulated ones, extracted from a two-level Rabi model (lines; see Appendix B for details). The simulated and measured mode profiles in Fig. 3(a) are, however, distorted compared to the mode profiles shown in Fig. 2(c), since the photon bandwidth $\Gamma=\kappa_{S}$ is comparable to both the interaction strength $g$ and the coupling rate $\kappa_{G}$ of the gate qubit to the transmission line. These distortions also lead to a finite imaginary part of the mode profiles of up to about $14 \%$ relative to the real part. During idle times, we do not parametrically drive the $|f 0\rangle \leftrightarrow|e 1\rangle$ transition, to ensure that the mode profile of a photonic qubit reflecting off of the gate qubit is independent of the state of the gate qubit. 


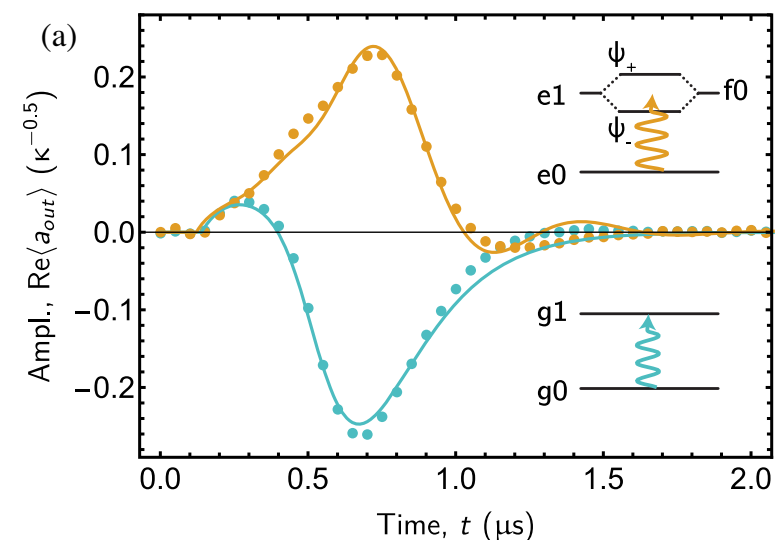

(b) Bell State, $F_{\text {ext }}=0.69$

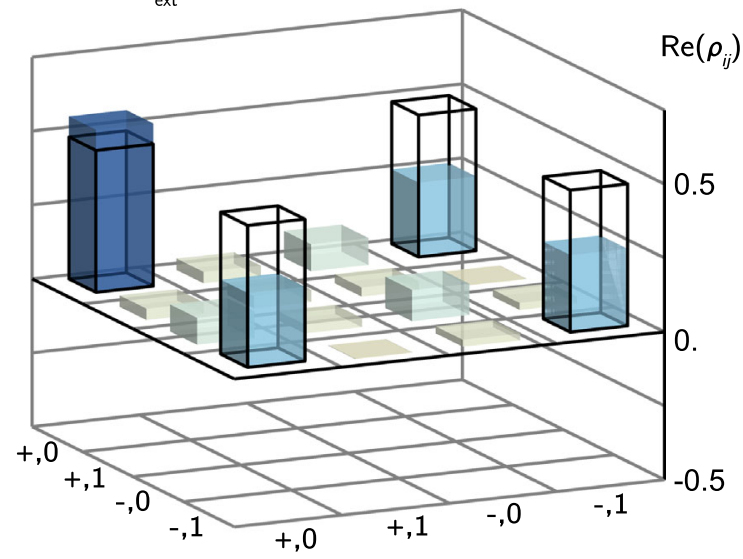

FIG. 3. (a) Measured (dots) and simulated (lines) real part of the envelope of the amplitude $\left\langle a_{\text {out }}\right\rangle$ for the states $|+\rangle$ emitted from the source qubit and reflected off of the gate qubit while driving the $|f 0\rangle \leftrightarrow|e 1\rangle$ transition of the gate qubit. Level diagrams and traces are shown for the gate qubit in the ground state (turquoise) and in the excited state (orange). The imaginary part (not shown) is below $0.04 / \sqrt{\kappa}$ in magnitude. (b) Real part of the measured density matrix $\rho_{\text {meas }}$ (solid bars) and the ideal density matrix $\rho_{\text {ideal }}=|\psi\rangle\langle\psi|$ (black wire frames) of the twomode Bell state $|\psi\rangle=(|+, 0\rangle+|-, 1\rangle) / \sqrt{2}$. The fidelity is $F=\left\langle\psi\left|\rho_{\text {meas }}\right| \psi\right\rangle=0.69$. Imaginary parts (not shown) are all below 0.04 in magnitude.

Having verified all basic ingredients, we demonstrate the entangling capability of the implemented photonic controlled-phase gate [Fig. 1(c); see Appendix C for the detailed pulse scheme] by generating a two-mode Bell state. To do so, we perform a controlled-phase gate between the two photonic modes $P_{1}$ and $P_{2}$, each prepared in the state $|+\rangle=(|0\rangle+|1\rangle) / \sqrt{2}$, measure the two field quadratures of both photonic qubits $P_{1}$ and $P_{2}$, and reconstruct the joint density matrix $\rho_{\text {meas }}[32,37]$, of which the real parts are shown in Fig. 3(b). We extract a fidelity of $F=\left\langle\psi\left|\rho_{\text {meas }}\right| \psi\right\rangle=0.69$ to the ideal state $|\psi\rangle=(|+, 0\rangle+$ $|-, 1\rangle) / \sqrt{2}$, mainly limited by photon loss, mostly from the circulator, contributing $13 \%$ to the infidelity, as well as decoherence and a finite absorption efficiency.
To further characterize the implemented single- and twoqubit gates, we perform quantum process tomography for identity, $X, Y$, and $T$ gates and the CPHASE gate, which together constitute a universal set of gates [41] acting on itinerant photonic qubits. We prepare photonic qubits in all cardinal states, perform the respective gate, and reconstruct the density matrix for each input state. We then extract the process maps $\chi_{\text {meas }}$ [42], shown in Fig. 4 assuming ideal input states, and calculate the process fidelities $F_{\text {tot }}=$ $\operatorname{Tr}\left(\sqrt{\sqrt{\chi_{\text {meas }}} \chi_{\text {ideal }} \sqrt{\chi_{\text {meas }}}}\right)^{2}$ with respect to the ideal process maps $\chi_{\text {ideal }}$. We obtain close to $75 \%$ fidelity for the singlequbit gates and $57 \%$ for the controlled-phase gate. The obtained fidelities are small compared to state-of-theart gate fidelities for stationary qubits directly coupled to each other on a single device [43-45], mainly due to the radiation loss in the circulator and due to decoherence during the process duration, which is on the order of a microsecond. The performance of the photonic single- and two-qubit gates could thus be greatly enhanced by the use of less lossy circulators [46,47] and by larger coupling rates.

To further analyze the obtained process maps and fidelities, we simulate the single-qubit gates using a master equation approach considering photon loss and decoherence, as well as truncation and finite bandwidth of the photonic mode (see Appendix D for details). The obtained, simulated process maps $\chi_{\text {sim }}$ have fidelities of around $97 \%$ to the measured process maps $\chi_{\text {meas }}$ and are thus in good agreement with $\chi_{\text {meas }}$. Therefore, the observed errors seem largely dominated by the aforementioned mechanisms, which we account for in the master equation simulations.

Having experimentally inferred and simulated process maps, we investigate the effect of state preparation errors, specifically photon loss, on the obtained fidelities, by characterizing the internal performance of the gate set. To do so, we apply the Kraus operators $K_{0}=|g\rangle\langle g|+$ $\sqrt{\eta_{\text {loss }}}|e\rangle\langle e|$ and $K_{1}=\sqrt{1-\eta_{\text {loss }}}|g\rangle\langle e|$ to all ideal input states, thereby generating effective input states affected by photon loss. We then extract the internal process map $\chi_{\text {int }}$ based on those effective input states and the measured density matrices (Fig. 4, blue wire frame). The resulting internal process fidelities $F_{\text {int }}=\operatorname{Tr}\left(\sqrt{\sqrt{\chi_{\text {int }}} \chi_{\text {ideal }} \sqrt{\chi_{\text {int }}}}\right)^{2}$ are close to $87 \%$ for single-qubit gates and $74 \%$ for the controlled-phase gate. Therefore, photon loss is the dominant error source for the presented gate set. Based on master equation simulations, we attribute most of the infidelity of the internal single-qubit processes to decoherence (13\%), while truncation of the photonic mode contributes only approximately $1 \%$; see Table I for a summary of different error sources. Extending the models presented in Appendix D to also simulate process fidelities of the two-qubit gate could be an interesting subject for future theoretical work. As a first step in this direction, 
(a) Id, $F=0.75,0.86$

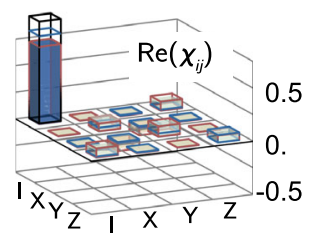

(c) $Y, F=0.76,0.87$

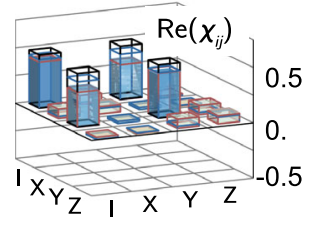

(b) $X, F=0.76,0.87$

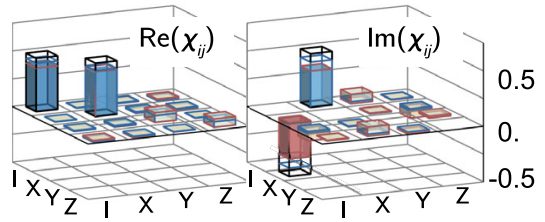

(d) $\mathrm{T}, F=0.76,0.87$

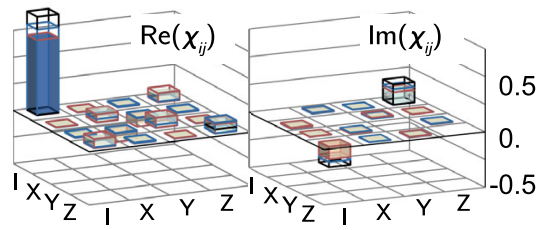

(e) CPHASE, $F=0.57,0.74$

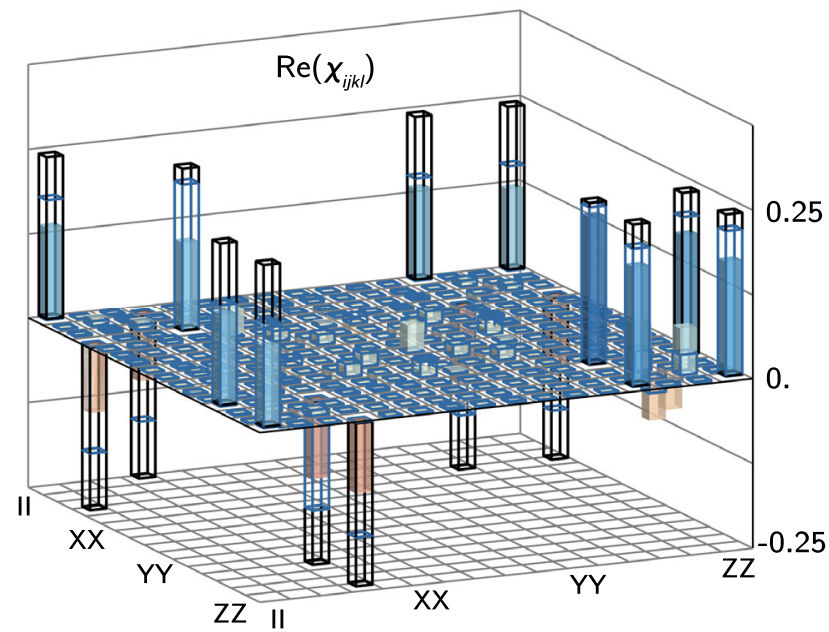

FIG. 4. Measured process maps with $\chi_{\text {int }}$ (blue wire frames) and without $\chi_{\text {meas }}$ (filled bars) accounting for state preparation errors for (a) the identity, (b) the $X$ gate, (c) the $Y$ gate, (d) the $T$ gate, and (e) the controlled-phase (CPHASE) gate. For comparison, we also show the simulated $\chi_{\text {sim }}$ (red wire frames) and ideal $\chi_{\text {ideal }}$ (black wire frames) process maps. $F=F_{\text {tot }}, F_{\text {int }}$, with the total fidelity $F_{\text {tot }}=$ $\operatorname{Tr}\left(\sqrt{\sqrt{\chi_{\text {meas }}} \chi_{\text {ideal }} \sqrt{\chi_{\text {meas }}}}\right)^{2}$ and the internal fidelity $F_{\text {int }}=\operatorname{Tr}\left(\sqrt{\sqrt{\chi_{\text {int }}} \chi_{\text {ideal }} \sqrt{\chi_{\text {int }}}}\right)^{2}$. Imaginary parts (not shown) are all below 0.04 in magnitude.

we estimate the effect of mode distortions encountered by the second photonic qubit when reflecting off of the gate chip during the two-qubit gate. To do so, we simulate the field amplitude $\psi_{g}\left(\psi_{e}\right)$ of a photonic qubit reflecting off of the gate qubit in the ground (excited) state. We then calculate the mode-matching efficiency $\eta_{F}=\left|\int \psi_{g}^{*} f(t) d t\right|^{2}$ [37], given by the overlap between $\psi_{g}$ and the chosen optimal filter function $f(t)=\left[\left(\psi_{g}-\right.\right.$ $\left.\left.\psi_{e}\right) /\left(\left\|\psi_{g}-\psi_{e}\right\|\right)\right]$ (see Appendix B for details). By treating the mode-matching inefficiency $1-\eta_{F}$, which for the parameters in our experiment amounts to $5 \%$, on similar grounds as the radiation loss, we estimate the corresponding gate infidelity to be on the order of $1-\eta_{F}$ (see Table I). The mode inefficiency $1-\eta_{F}$ depends on the interaction strength $g$, the coupling rate $\kappa$, and the photon bandwidth $\Gamma$ and vanishes in the limit of $g / \Gamma \approx \kappa / \Gamma \gg 1$. By increasing $g / \Gamma \approx \kappa / \Gamma$ in future experiments, we thus expect that the effect of mode distortions on the process fidelity of the controlled-phase gate can be greatly reduced.

TABLE I. Relative contribution of decoherence, radiation loss, and mode distortion to the total process fidelity resulting from simulations described in the text. The contribution of decoherence to the two-qubit process fidelity is estimated from the measured process fidelity and the simulated values of the other two contributions. The total fidelity is given by the product of the relative contributions.

\begin{tabular}{lccc}
\hline \hline & Decoherence & Radiation loss & Mode distortion \\
\hline Single-qubit & 0.87 & 0.86 & 0.99 \\
Two-qubit & 0.78 & 0.77 & 0.95 \\
\hline \hline
\end{tabular}

In summary, we implement a universal gate set for itinerant, microwave photonic qubits. The observed internal process fidelities are close to $87 \%$ for singlequbit gates, limited mainly by decoherence, and close to $74 \%$ for the controlled-phase gate. The total processes, however, are still limited by photon loss. In future, this may be overcome by the development of less lossy circulators $[46,47]$ or by heralding of photons using timebin [24,48], frequency-comb [23], or dual-rail encoding [21]. We expect the success rate of such heralding schemes to be an order of magnitude larger (close to $\eta_{\text {loss }}=75 \%$ ) than in optical implementations of deterministic photon-photon gates [30,31]. In addition, increasing the photon bandwidth $\Gamma$ by engineering larger coupling rates between stationary qubits and photonic qubits may reduce errors due to decoherence. We envision the demonstrated gate set to be used to generate larger entangled many-body quantum states, for example, by creating additional entanglement bonds between photonic qubits $[49,50]$.

The data produced in this work are available from the corresponding authors upon reasonable request.

\section{ACKNOWLEDGMENTS}

We thank Mihai Gabureac for contributions to the device fabrication, Baptiste Royer for input on the manuscript and the master equation simulations, and Gerhard Rempe for comments on the manuscript. This work was supported by the Swiss National Science Foundation (SNSF) through the project "Quantum Photonics with Microwaves in Superconducting Circuits" (Grant 
No. 200021_184686), by the European Research Council (ERC) through the project "Superconducting Quantum Networks" (SuperQuNet), by the National Centre of Competence in Research "Quantum Science and Technology" (NCCR QSIT), a research instrument of the Swiss National Science Foundation (SNSF), by the European Unions Horizon 2020 research and innovation program under Grant Agreement No. 899354 (SuperQuLAN), and by ETH Zurich. K. R. and J.-C. B. designed the device. J.-C.B. and G. J. N. fabricated the device. K. R. and J.-C. B. prepared the experimental setup. K. R. and J.-C. B. characterized and calibrated the device and the experimental setup. L. W., P. M., and P. K. implemented the photon shaping. K. R., J.-C.B., and L.W. carried out the experiments and analyzed the data, with support from P. M. and P. K. K. R. and C. E. wrote the manuscript with input from all coauthors. A. W. and C. E. supervised the work.

The authors declare no competing interests.

\section{APPENDIX A: EXPERIMENTAL SETUP AND BASIC CALIBRATIONS}

We fabricate two $4.3 \mathrm{~mm} \times 7 \mathrm{~mm}$ samples, both based on the same base layer design - one for the source qubit and one for the gate qubit. The sample for the gate qubit is also used in Ref. [32]; see Fig. 5 for a false color optical micrograph. Using optical photolithography and reactive ion etching, we pattern qubits pads and coplanar waveguide structures into a niobium film sputtered on a silicon substrate. To fabricate the $\mathrm{Al} / \mathrm{AlOx} / \mathrm{Al}$ Josephson junctions, we use electron-beam lithography and shadow evaporation. We mount the packaged samples onto the base temperature stage $(20 \mathrm{mK})$ of a dilution refrigerator, each housed inside its dedicated set of magnetic shields made of aluminum and cryoperm; see the sketch of the experimental setup in Fig. 6. The two samples are connected via coaxial cables and a circulator, which is also thermalized to the base plate. We apply microwave pulses to the source and gate qubit and to the respective readout resonators via charge lines incorporating $20 \mathrm{~dB}$ of attenuation at the $4 \mathrm{~K}, 100 \mathrm{mK}$, and base temperature stage for signal conditioning [51]. We use phase-preserving Josephson parametric amplifiers (JPAs) [52] to amplify both the gate-qubit-readout signal and the field associated with the photonic qubits with a gain of 25 and $14 \mathrm{~dB}$, respectively. The JPA for the source-qubit readout is not used. All JPAs are mounted in a pair of cryoperm shields. We further amplify the detection signals using a high-electron mobility transistor at the $4 \mathrm{~K}$ stage and room-temperature amplifiers. Compared to the readout lines, the photon detection line has one amplification stage less to preserve the linearity in the amplification chain, which is a crucial requirement for photon field tomography [37].

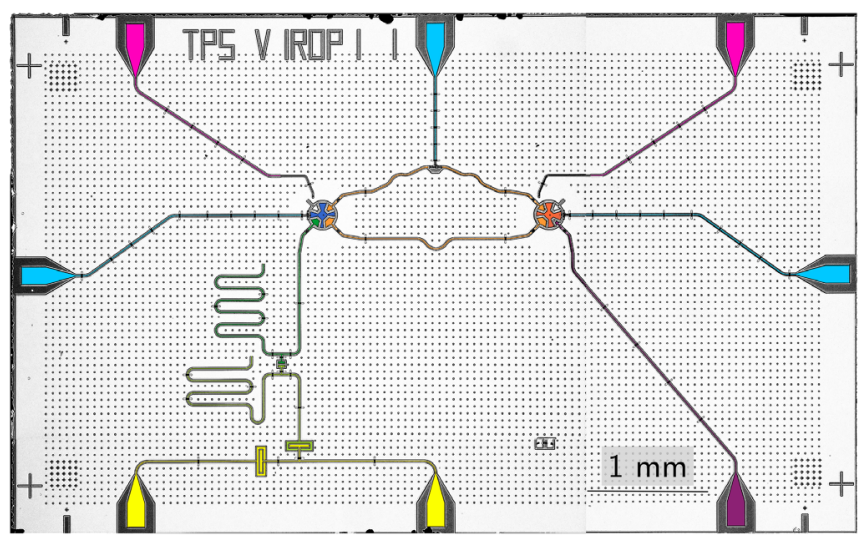

FIG. 5. False color optical micrograph of the sample of the gate qubit (blue). Depicted are the two coupler paths (orange) and the converter mode (dark orange), coupled to the transmission line (purple). Flux lines (cyan) and charge lines (pink) couple to the gate qubit, converter mode, and the coupler. The readout resonator (green) is coupled to a feed line (yellow) via a Purcell filter (light green).

We measure the basic device parameters presented in Table II, by performing one- and two-tone spectroscopy measurements, as well as Rabi, Ramsey, and coherence measurements, as presented in Ref. [32]. Both the gate and the source qubit are tuned to their respective maximum frequency [53]. Their comparably short dephasing times are possibly limited by magnetic flux noise, which could be further suppressed in the future by integrating additional magnetic shielding. The converter modes are both tuned to $\omega_{01} / 2 \pi=5.998 \mathrm{GHz}$.

To protect the source (gate) qubit from Purcell decay via the converter mode into the transmission line, we mediate the coupling between the converter mode and the source (gate) qubit by two coupling resonators. One of the two coupling resonators is tunable by magnetic flux applied to the SQUID embedded in the resonator. The other coupling resonator is realized as a coplanar waveguide resonator with a fundamental mode frequency of about $29 \mathrm{GHz}$ and mediates a static coupling of about $13 \mathrm{MHz}$. Since the frequency of the tunable resonator (tunable between approximately 1 and approximately $4.9 \mathrm{GHz}$ for both the source and the gate qubit) is lower than the qubit frequency, the coupling provided by the tunable and the static resonator is of opposite sign [43]. Therefore, we bias the tunable nonlinear resonator to the point at which the static coupling is canceled, such that the effective coupling between the source or gate qubit and the converter mode $J$ vanishes in the absence of parametric driving fields. Experimentally, we estimate an upper bound for the effective coupling $J$ at the cancellation point by tuning the converter mode into resonance with the qubit and measuring the qubit lifetime $T_{1}$. We obtain an upper bound $J / 2 \pi \leq \sqrt{\kappa /\left(4 T_{1, \text { Purc }}\right)} / 2 \pi \approx 20 \mathrm{kHz}$, with 


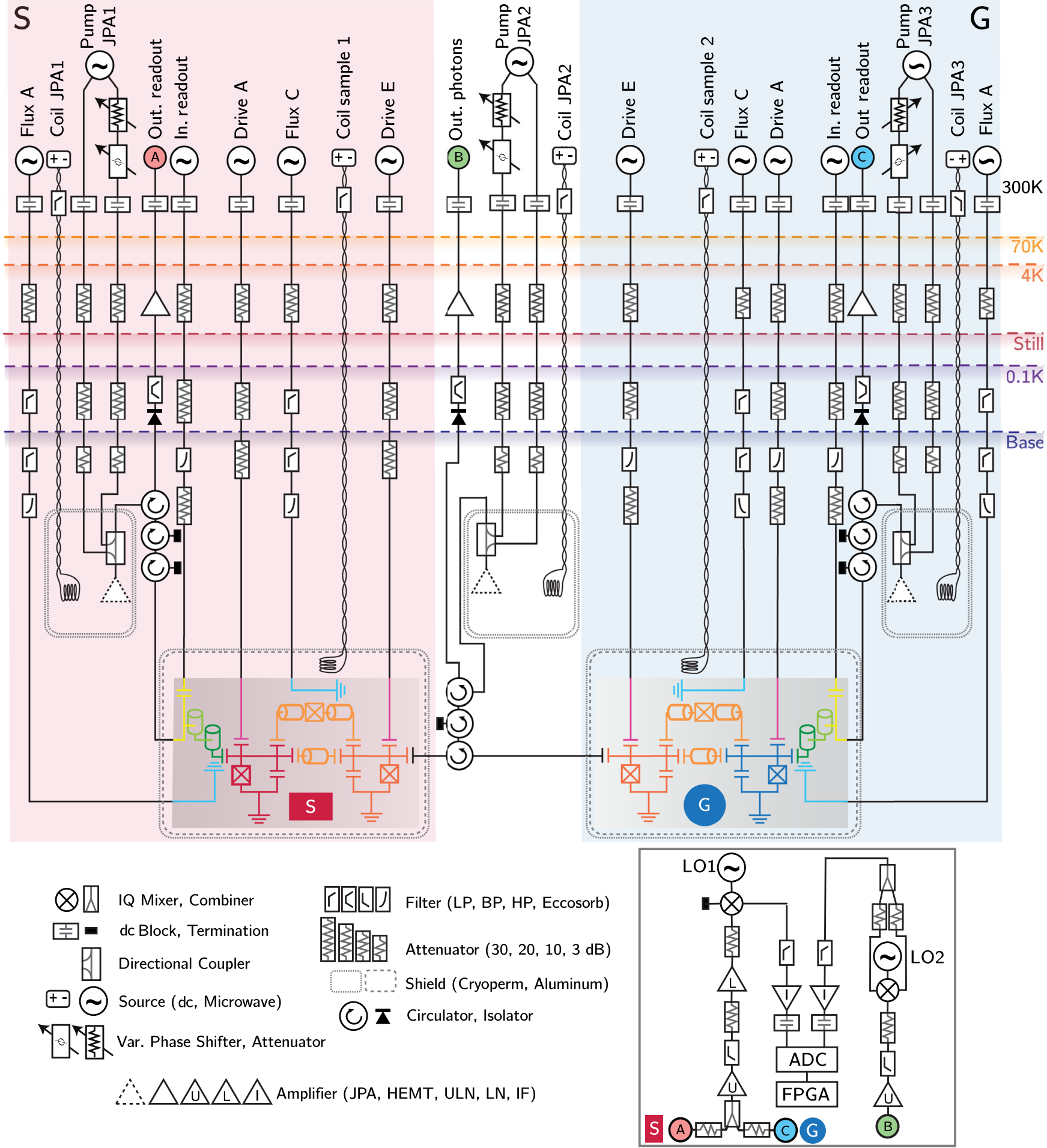

FIG. 6. Experimental setup for operating the source qubit $(S$, red) and the gate qubit ( $G$, blue) devices. For details, see the text.

$1 / T_{1, \text { Purc }}=1 / T_{1}-1 / T_{1, \text { Int }}$, assuming an internal lifetime $T_{1, \text { Int }}=20 \mu \mathrm{s}$.

We extract the decay rate $\kappa$ for the respective converter modes by measuring the coherent scattering of a weak input one into the transmission line vs frequency and by fitting the data to the function $\left|S_{21}\right|=\left|S_{0} /(1+2 i \delta 2 \pi / \kappa)\right|$, obtained from input-output theory with $\delta$ being the frequency detuning between the converter mode frequency $\omega_{01} / 2 \pi$ and the probe frequency and $S_{0}$ being the transmission at zero detuning; see Fig. 7. 
TABLE II. Measured device parameters.

\begin{tabular}{|c|c|c|c|}
\hline & & Source & Gate \\
\hline \multirow[t]{7}{*}{ Qubit } & $g$-e frequency, $\omega_{g e} / 2 \pi[\mathrm{GHz}]$ & 5.925 & 5.771 \\
\hline & $e-f$ frequency, $\omega_{e f} / 2 \pi[\mathrm{GHz}]$ & 5.630 & 5.478 \\
\hline & Anharmonicity, $\alpha / 2 \pi[\mathrm{MHz}]$ & 295.0 & 301.7 \\
\hline & Lifetime of $|e\rangle, T_{1}^{(\bar{e})}[\mu \mathrm{s}]$ & 16 & 13 \\
\hline & Lifetime of $|f\rangle, T_{1}^{(f)}[\mu \mathrm{s}]$ & 6 & 4 \\
\hline & Dephasing time of $|e\rangle, T_{2}^{\star(e)}[\mu \mathrm{s}]$ & 4 & 10 \\
\hline & Dephasing time of $|f\rangle, T_{2}^{\star(f)}[\mu \mathrm{s}]$ & 2 & 2 \\
\hline Coupler & Frequency, $\omega_{\mathrm{c}} / 2 \pi[\mathrm{GHz}]$ & 3.2 & 4.6 \\
\hline \multirow[t]{2}{*}{ Converter } & $0-1$ frequency, $\omega_{01} / 2 \pi[\mathrm{GHz}]$ & 5.998 & 5.998 \\
\hline & Decay rate, $\kappa / 2 \pi[\mathrm{MHz}]$ & 1.8 & 2.1 \\
\hline
\end{tabular}

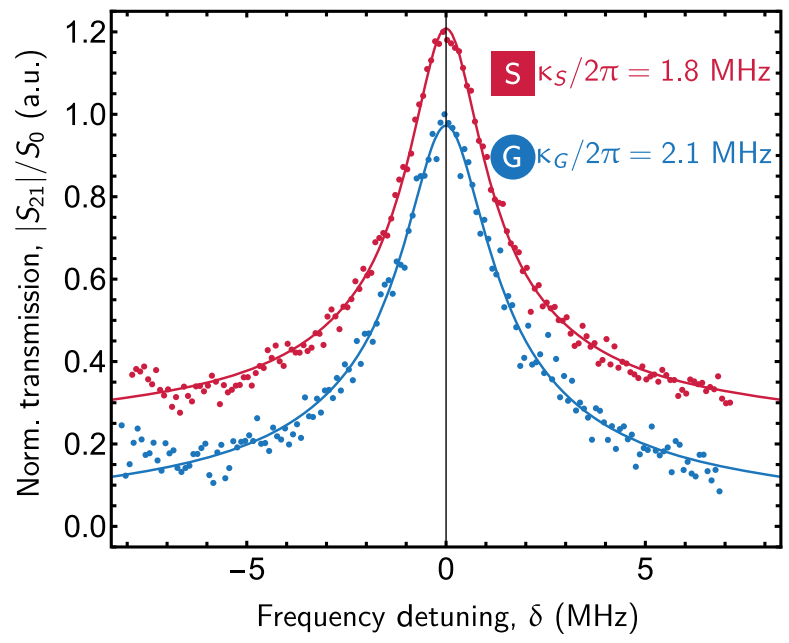

FIG. 7. Absolute value of the measured transmission coefficient $\left|S_{21}\right| / S_{0}$, normalized to the transmission at zero detuning $S_{0}$, when driving the converter mode of the gate qubit (blue) and the source qubit (red, vertically offset by 0.2 ) via their respective charge lines. Solid lines are Lorentzian fits to the data (see the text), resulting in the indicated linewidths $\kappa$.
To calibrate the power of the emitted photonic qubits, we measure the incoherent scattering while driving the converter mode resonantly at the set of indicated drive powers, observing Mollow triplets; see Fig. 8. We globally fit the observed data to the expected power spectral density function $P$ vs the detuning from the drive frequency $\delta$, obtained by considering a dressed two-level system $[54,55]$ :

$$
\begin{aligned}
P(\delta) & =P_{0} \kappa \frac{64 \kappa \Omega^{4}\left[2 \kappa^{2}+2\left(\delta-f_{0}\right)^{2}+\Omega^{2}\right]}{\pi\left[\kappa^{2}+4\left(\delta-f_{0}\right)^{2}\right]\left(\kappa^{2}+2 \Omega^{2}\right) p_{+} p_{-}}, \quad \text { with } \\
p_{ \pm} & =\left[5 \kappa^{2}+8\left(\delta-f_{0}\right)^{2}-8 \Omega^{2} \pm 3 \kappa \sqrt{\kappa^{2}-16 \Omega^{2}}\right]
\end{aligned}
$$

where $\kappa=\kappa_{S / G}$ is the decay rate of the respective converter mode, $\Omega$ is the drive rate, and $f_{0}$ takes into account small detunings between the drive frequency and the converter mode. From the fitted amplitudes $P_{0}$ for the source device $P_{0, S}$ and the gate device $P_{0, G}$, we extract the transmission efficiency expected from radiation loss between the source and the gate chip of $\eta_{\text {loss }}=P_{0, S} / P_{0, G}=75 \%$.

\section{APPENDIX B: PHOTON SHAPING}

\section{Concept}

To optimize the absorption efficiency, we generate photonic fields with a near time-symmetric temporal profile by controlling the time evolution of the tunable, effective coupling rate $J$ between the qubit and its converter mode, following the protocol detailed in Refs. [38-40]. We choose the temporal envelope

$$
\xi(t)=\frac{\sqrt{\Gamma}}{2} \operatorname{sech}(\Gamma t / 2),
$$

(b)

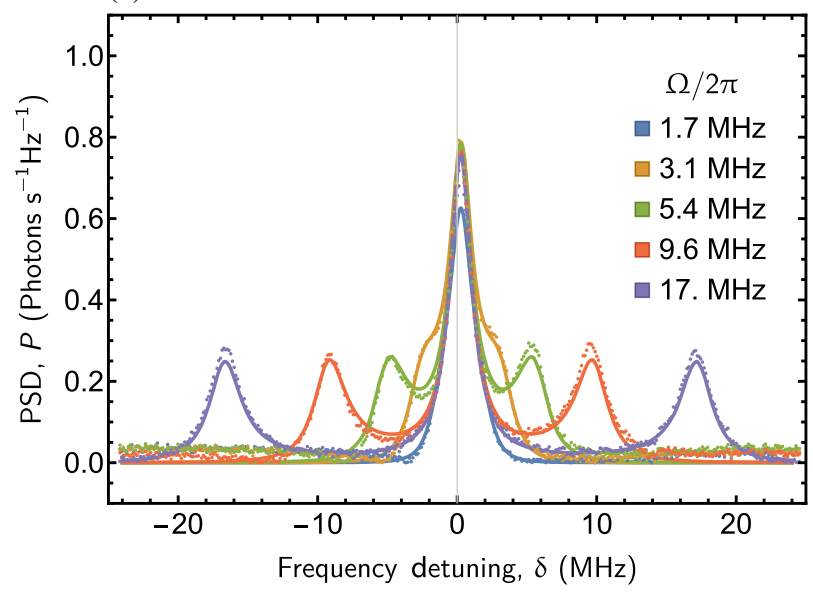

FIG. 8. Measured power spectral density (PSD) $P$ of the inelastic scattering of a coherent tone resonant with (a) the source qubit and (b) the gate qubit converter mode for the indicated drive rates $\Omega$. All datasets are scaled with respect to the gate qubit fitted amplitude $P_{0, G}$; see the text. Solid lines are joined fits to all datasets with a single set of fitting parameters. The drive rates $\Omega$ are fitted independently for each dataset. 
where the effective bandwidth $\Gamma$ is bound by the coupling rate $\kappa$ of the converter mode: $\Gamma \leq \kappa$. To emit photonic fields with the temporal envelope $\xi(t)$, the effective coupling rate is controlled according to [38]

$$
J(t)=\frac{\Gamma\left[-e^{\Gamma t}+1+\kappa\left(1+e^{\Gamma t}\right) / \Gamma\right]}{4 \cdot \cosh (\Gamma t / 2) \sqrt{\left(1+e^{\Gamma t}\right) \kappa / \Gamma-e^{\Gamma t}}} .
$$

To controllably absorb photonic fields with an envelope $\xi(t)$, we apply a time-reversed coupling $J(-t)$.

We choose to emit shaped photons with the maximal effective bandwidth $\Gamma$, thereby minimizing errors due to decoherence. Since we emit photonic qubits from the source qubit $S$ and absorb them at the gate qubit $G, \Gamma$ is limited by the smaller coupling rate of the two converter modes $\Gamma \leq \min \left[\kappa_{S}, \kappa_{G}\right]$. In our setup, $\kappa_{S}<\kappa_{G}$. Therefore, the effective photon bandwidth $\Gamma$ is limited by the coupling rate $\kappa_{S}$ of the source qubit converter mode.

\section{Implementation}

We control the time evolution of the effective coupling rate $J$ by applying an alternating current (ac) pulse $I(t)$ to the flux line of the respective coupler. We generate the ac pulse $I(t)$ using an arbitrary waveform generator (AWG). We combine the ac pulse with the direct current (dc) offset applied for biasing the coupler and route the combined pulse into the coupler flux line. The ac pulse $I(t)$ induces an alternating magnetic flux $\Phi(t)$ in the SQUID loop of the coupler. As a result, the coupler frequency oscillates at the frequency of the ac pulse $I(t)$. When the ac pulse $I(t)$ is in resonance with the $|e 0\rangle \leftrightarrow|g 1\rangle$ transition frequency, we control the amplitude and the phase of the effective coupling rate $J(t)$.

To tune the ac pulse into resonance with the $|e 0\rangle \leftrightarrow|g 1\rangle$ transition frequency, we prepare the qubit in the excited state and measure the population remaining after applying a modulated square pulse $I_{\square}(t)=A \cos (\omega t)[\Theta(0)-\Theta(\tau)]$ with varying frequency $\omega / 2 \pi$ [see Fig. $9($ a) $]$ for a range of pulse amplitudes $A$ at a fixed pulse duration $\tau$. $\Theta(t)$ indicates the Heaviside step function. We determine the frequency of the $|e 0\rangle \leftrightarrow|g 1\rangle$ transition as the one for which the excited state population is minimized. Since we drive an excitation-number-conserving transition via a tunable coupler, we avoid ac Stark effects as compared to the scheme in Refs. [38,39].

To measure the effective coupling rate $J$, we vary the duration $\tau$ of the square pulse $I_{\square}(t)$ at fixed, resonant frequency $\omega / 2 \pi$ [see Fig. 9(b)] for $A=0.35$. By considering a driven two-level system decaying into the ground state with rate $\kappa$ [38], we fit the measured population vs duration $\tau$ to obtain the effective coupling rate $J$. From the same fit, we also extract the coupling rates of the converter modes to the transmission line $\kappa_{S} / 2 \pi=2.1 \mathrm{MHz}$ and $\kappa_{G} / 2 \pi=2.8 \mathrm{MHz}$, which deviate from the coupling rates obtained by spectroscopy (Fig. 7). The nature of this deviation is yet to be investigated, but simulations show that the relative impact on the gate fidelities is below $2 \%$. We use the fitted coupling rates $\kappa_{S} / 2 \pi=2.1 \mathrm{MHz}$ and $\kappa_{G} / 2 \pi=2.8 \mathrm{MHz}$ for the generation of coupler pulses $I_{\xi}(t)$. Finally, we obtain a mapping between the effective coupling rate $J$ and the amplitude of the square pulse $A$ by interpolating the fitted coupling rates vs amplitude $A$ with an effective model, considering first-order (linear) and second-order (quadratic) terms, shown in Fig. 9(c). For the (a)

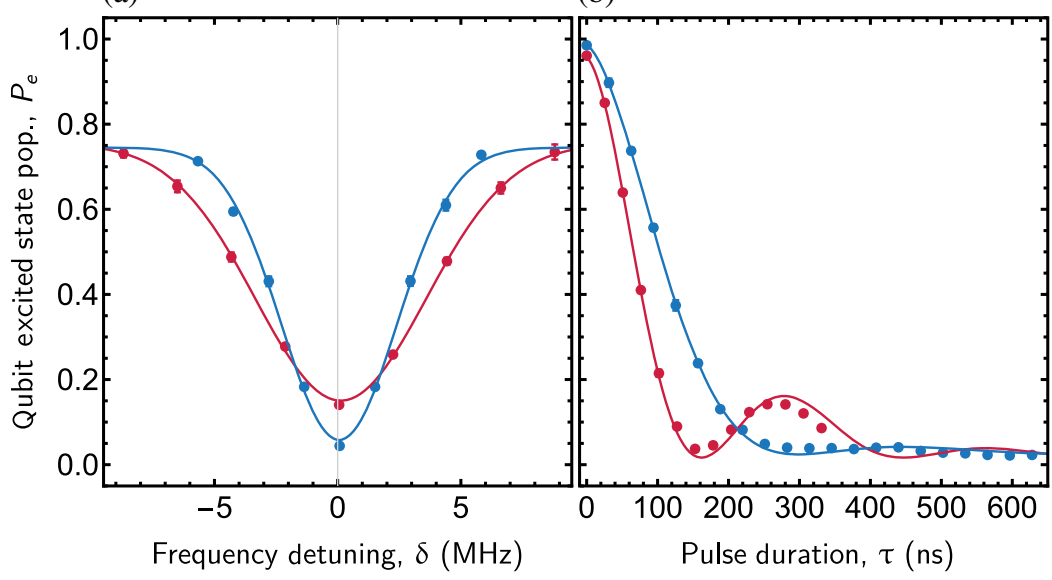

(c)

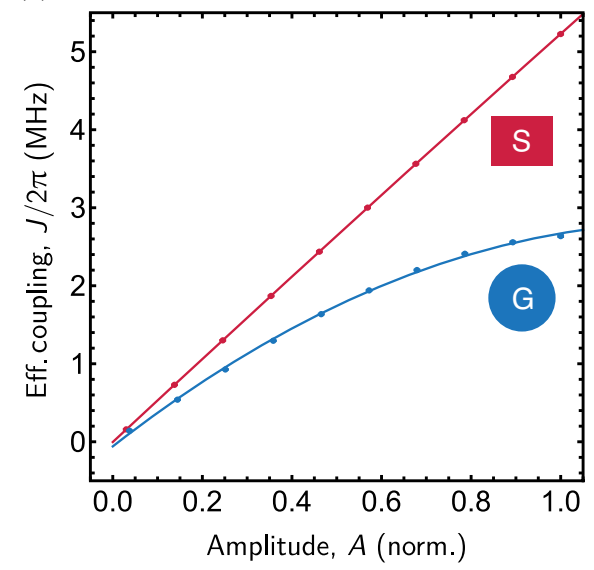

FIG. 9. (a),(b) Excited state population $P_{e}$ after preparing the source qubit (red) [gate qubit (blue)] in the excited state and applying a modulated flux pulse with a square envelope and a fixed, normalized amplitude of $A=0.35$. (a) Fixed pulse duration $\tau=283$ ns (349 ns), plotted vs flux pulse frequency detuning $\delta$ from the $|e 0\rangle \leftrightarrow|g 1\rangle$ transition. Solid lines are Gaussian fits to the data. (b) On resonance $\delta=0$, plotted vs flux pulse duration $\tau$. Solid lines are fits to the data; see the text. (c) Effective coupling rate $J$ for the $|e 0\rangle \leftrightarrow|g 1\rangle$ transition of the gate qubit $(G$, blue circle) and the source qubit ( $S$, red rectangle) vs drive amplitudes $A$, normalized to the respective maximal amplitude. Solid lines are quadratic fits to data. 
gate qubit, we observe that maximum coupling rate $J$ is reached for smaller flux pulse amplitudes, since the gate qubit coupler is biased closer to its maximum frequency. By inverting the mapping $A \rightarrow J$, we convert the targeted time evolution of the coupling rate $J(t)$ in Eq. (B2) into a coupler pulse $I_{\xi}(t)$ that is generated by the AWG.

To minimize the effects of decoherence, we truncate the coupler pulse $I_{\xi}(t)$ at $t= \pm 4.6 / \Gamma$. We estimate that the photon shape generated by the truncated pulse has an overlap of $98 \%$ with the targeted envelope. For the estimate, we numerically evaluate a two-level Rabi model decaying into the ground state with rate $\kappa$ while being driven by the coupler pulse $I_{\xi}(t)$. The overlap provides a good estimate of the absorption efficiency $[38,40]$. The two-level Rabi model is also used to extract the expected temporal profiles shown as solid lines in Fig. 2(c). To extract the expected temporal profiles after reflection of a photonic mode off the gate qubit [shown in Fig. 3(a)], we calculate the reflection coefficient $S_{11}$ vs frequency of the gate device while driving the $|f 0\rangle \leftrightarrow|e 1\rangle$ transition, for the gate qubit in the ground state $|g\rangle$ and excited state $|e\rangle$ using input-output theory. We then convolve the temporal profile of the incoming photonic mode with the response function for the gate qubit in the ground state $|g\rangle$ and excited state $|e\rangle$, obtained by Fourier transforming the respective spectrum $S_{11}$. From the temporal profiles after reflection off of the gate qubit in the ground state $\psi_{g}$ and in the excited state $\psi_{e}$, we extract the mode-matching efficiency $\eta_{F}=\left|\int \psi_{g}^{*} f(t) d t\right|^{2}$ [37] of $95 \%$. To maximize the mode-matching efficiency for both $\psi_{q}$ and $\psi_{e}$, we choose the filter function $f(t)=$ $\left[\left(\psi_{g}-\psi_{e}\right) /\left(\left\|\psi_{g}-\psi_{e}\right\|\right)\right]$, such that $\eta_{F}=\left|\int \psi_{g}^{*} f(t) d t\right|^{2}=$ $\left|\int \psi_{e}^{*} f(t) d t\right|^{2}$. Note that, to obtain the filter function for reconstructing the density matrices, we use the measured temporal profiles after reflection off of the gate qubit in the ground state or the excited state [see Fig. 3(a)] instead of the simulated ones.

In addition, we simulate the mode-matching efficiency $\eta_{F}$ for different interaction strengths $g$ and find that the efficiency $\eta_{F}$ is maximized around $g / 2 \pi=1.5 \mathrm{MHz}$, which is close to the value used in the experiment $g / 2 \pi=$ $1.6 \mathrm{MHz}$ (see Appendix C). We also investigate the effect of mode distortions for larger coupling rates (which enable faster photon emission and absorption), by simulating the mode-matching efficiency for different interaction strengths $g$, coupling rates $\kappa$, and photon bandwidths $\Gamma$, assuming photons with temporal profile $\xi(t)=(\sqrt{\Gamma} / 2) \operatorname{sech}(\Gamma t / 2)$. We find the mode-matching efficiency $\eta_{F}$ to depend only on $g / \Gamma$ and $\kappa / \Gamma$ and to approach unity in the limit of $g / \Gamma \approx \kappa / \Gamma \gg 1$ (see Fig. 10) for $\kappa=g / 0.7$. Therefore, if we increase the coupling rates $\kappa \sim J \sim g$, we can emit photons with larger bandwidth $\Gamma$ and thus expect to reduce the infidelity of the controlledphase gate due to decoherence, and/or we can increase $g / \Gamma$ and $\kappa / \Gamma$ and thereby expect to reduce the infidelity due to

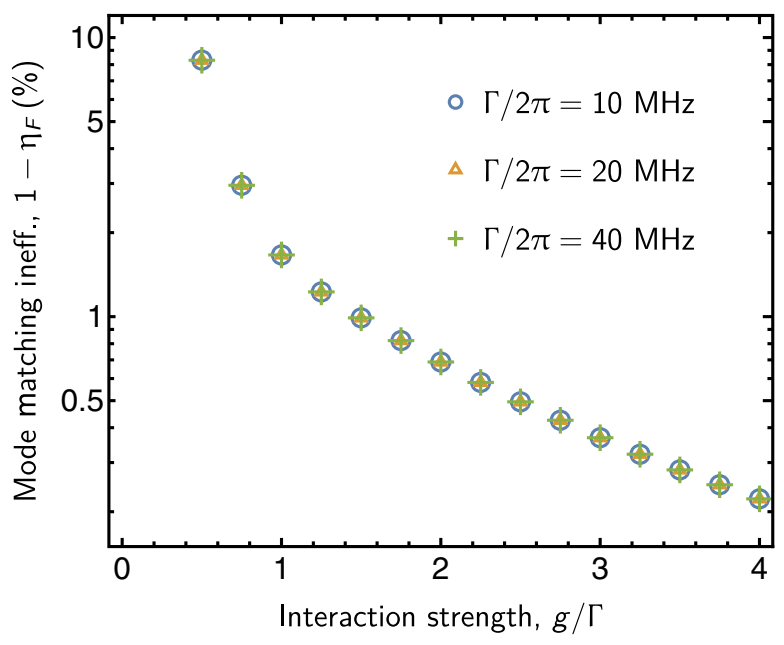

FIG. 10. Simulated mode-matching inefficiency $1-\eta_{F}=1-$ $\left|\int \psi_{g}^{*} f(t) d t\right|^{2}$ of time-symmetric photonic qubits with shape $\xi(t)=(\sqrt{\Gamma} / 2) \operatorname{sech}(\Gamma t / 2)$ reflected off of the gate qubit in the ground $\psi_{g}$ or the excited state $\psi_{e}$ vs interaction strength normalized by the photon bandwidth $g / \Gamma$, for indicated photon bandwidths $\Gamma$ and a coupling rate $\kappa=g / 0.7$. As a filter function for mode matching, we use $f(t)=\left[\left(\psi_{g}-\psi_{e}\right) /\left(\left\|\psi_{g}-\psi_{e}\right\|\right)\right]$.

mode distortions. Experimentally verifying that larger coupling rates reduce the effect of decoherence and/or mode distortions on the performance of the controlledphase gate would be an interesting subject for future experimental work.

\section{Single-photon tomography}

To verify the single-photon character of the photonic qubits, we prepare the source and the gate qubit in the state $\sin (\theta / 2)|0\rangle+\cos (\theta / 2)|1\rangle$ for the indicated range of angles $\theta$ and apply a swap gate using the coupler pulse $I_{\xi}(t)$. We measure the generated photonic qubits using heterodyne detection and extract moments up to fourth order [37] (shown in Fig. 11) normalized by the value of $\left\langle a^{\dagger} a\right\rangle$ for $\theta=\pi$ for the gate qubit. For both qubits, we find that $\left\langle\left(a^{\dagger}\right)^{2} a^{2}\right\rangle$ is close to zero but slightly negative. While we do not study the origin of this effect in detail, a weak gain compression in the amplification chain on the order of $0.01 \mathrm{~dB}$ caused by the single-photon signal at its input could explain the observed negative values in the inferred $\left\langle\left(a^{\dagger}\right)^{2} a^{2}\right\rangle$ correlations. The amplitude $\operatorname{Re}\langle a\rangle$ is reduced, compared to the ideal value, due to dephasing during the emission process. The source qubit has a smaller dephasing time $\left(T_{2, S}^{\star(e)}=4 \mu \mathrm{s}\right)$ than the gate qubit $\left(T_{2, G}^{\star(e)}=10 \mu \mathrm{s}\right)$; thus, the reduction in the amplitude $\operatorname{Re}\langle a\rangle$ is more pronounced. Error bars are obtained by evaluating five independent measurements. Moments $\left\langle\left(a^{\dagger}\right)^{n} a^{m}\right\rangle$ for the source qubit are reduced by a factor of $\eta_{\text {loss }}^{(n+m) / 2}=$ $0.75^{(n+m) / 2}$ due to photon loss between the source and the gate qubit. 
(a)

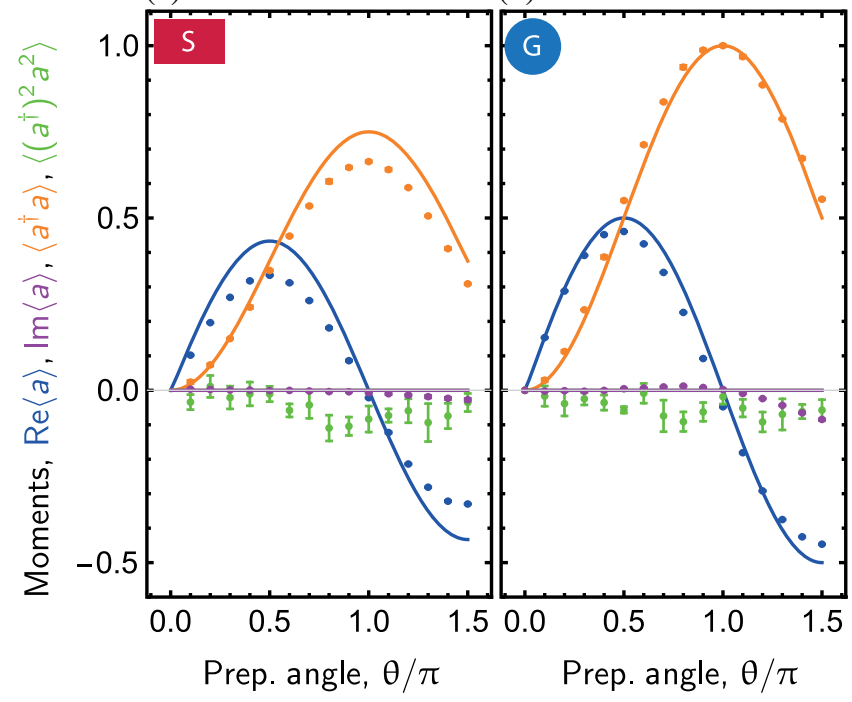

FIG. 11. Measured moments $\operatorname{Re}\langle a\rangle$ (blue), $\operatorname{Im}\langle a\rangle$ (purple), $\left\langle a^{\dagger} a\right\rangle$ (orange), and $\left\langle\left(a^{\dagger}\right)^{2} a^{2}\right\rangle$ (green), for superpositions $|\psi\rangle=$ $\sin (\theta / 2)|0\rangle+\cos (\theta / 2)|1\rangle$ of vacuum and single-photon states emitted from (a) the source qubit and (b) the gate qubit. Lines indicate ideal expectation values, calculated taking into account photon loss between the source and the gate chip.

\section{APPENDIX C: PHOTON GATE CALIBRATION AND PULSE SCHEME}

Having calibrated the emission process for both source and gate qubit individually, we optimize the simultaneous emission and absorption process to maximize the absorption efficiency. Specifically, we emit a single-photon Fock state $|1\rangle$ from the source qubit, absorb it at the gate qubit, and read out the state of the gate qubit. We repeat this transfer scheme while sweeping the experimental parameters detailed hereafter.

First, to ensure that both converter modes are exactly in resonance, we maximize the excited state population at the gate qubit while sweeping the gate qubit converter mode frequency; see Fig. 12(a). For each converter mode frequency, we repeat the calibrations for emitting and absorbing shaped photons, as detailed in Appendix B. Second, we calibrate the timing of pulses by sweeping the delay between emission and absorption pulses, shown in Fig. 12(b). For the optimal time delay, the excited state population is again maximal and sets the optimized transfer efficiency of $64 \%$. Third, we calibrate the unknown phase offset, originating from the optical path length between the two chips. To do so, we emit and absorb a superposition state $\left(|0\rangle+e^{i \varphi}|1\rangle\right) / \sqrt{2}$ sweeping the phase $\varphi$, apply a $\pi / 2$ pulse to the gate qubit, and extract the excited state populations at the gate qubit $G$, shown in Fig. 12(c). Master equation simulations (see Appendix D) are in good agreement with the measured calibration datasets.

Subsequently, we implement the universal gate set, consisting of the identity, the $X$, the $Y$, the $T$, and the controlled-phase gate in our setup using the pulse schemes shown in Fig. 13(a) for single-photon gates and Fig. 13(b) for the photon-photon gate. Coupler pulses $I_{\xi}(t)$ for emission and absorption (green in Fig. 13) are applied to the respective coupler. We realize $X$ and $Y$ gates on the transmon qubits by using derivative removal by adiabatic gate (DRAG) pulses [56] with amplitude $\theta=\pi / 2$ and a phase of $\varphi=\pi / 2$ and $\varphi=0$, respectively, truncated at $\pm 3 \sigma$, where $\sigma=6 \mathrm{~ns}$ is the linewidth of the pulse. The identity gate is realized by waiting $t=36 \mathrm{~ns}$, to compensate for the time required to perform the $X$ and the $Y$ gate on

(a)

(b)

(c)

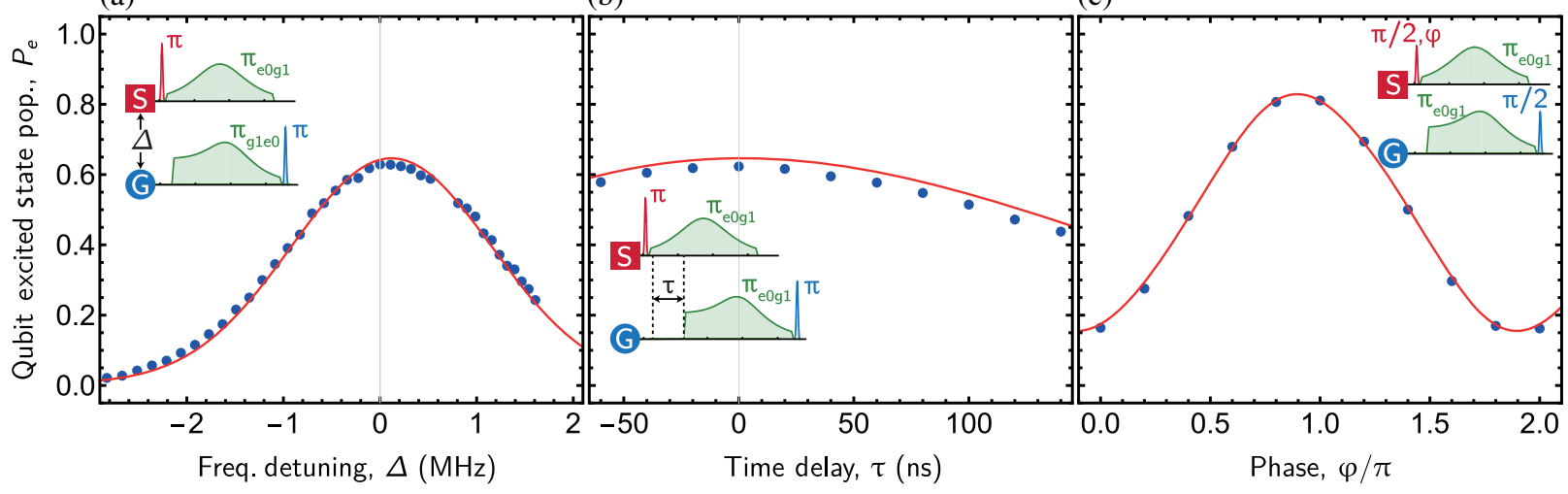

FIG. 12. Measured (blue dots) and simulated (red line) excited state population $P_{e}$ of the gate qubit for three calibration routines. The inset shows the envelopes of the pulse sequence applied to the gate chip ( $G$, blue circle) and the source chip $(S$, red rectangle) during the respective calibration steps. (a) Measured excited state population $P_{e}$ after emitting a single-photon Fock state $|1\rangle$ from the source qubit and absorbing it at the gate qubit vs frequency detuning $\Delta$ between the converter modes of the source and gate qubit. Photons are emitted or absorbed in resonance with the respective converter mode. (b) Excited state population $P_{e}$ vs time delay $\tau$ (relative to the optimal time delay found in the experiment) between the emission pulse at the source qubit and the absorption pulse at the gate qubit. (c) Excited state population $P_{e}$, when emitting and absorbing a photonic superposition state $\left(|0\rangle+e^{i \varphi}|1\rangle\right) / \sqrt{2}$ vs phase $\varphi$ and applying a final $\pi / 2$ rotation along the $Y$ axis to the gate qubit. 
(a) $\mathrm{S}$

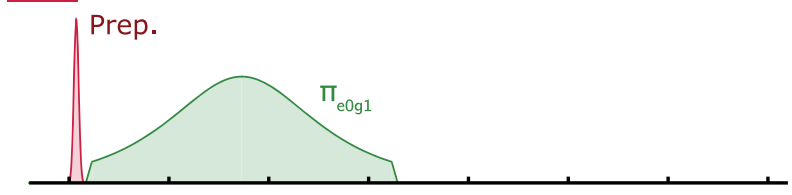

G

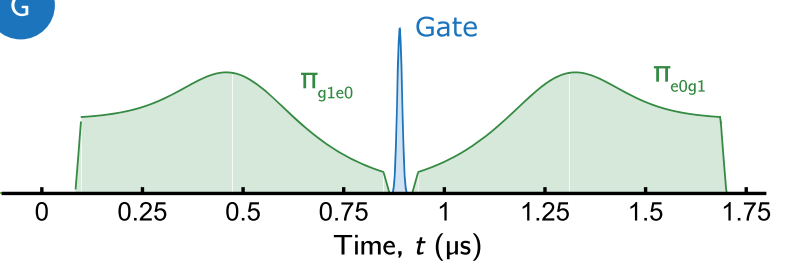

(b)

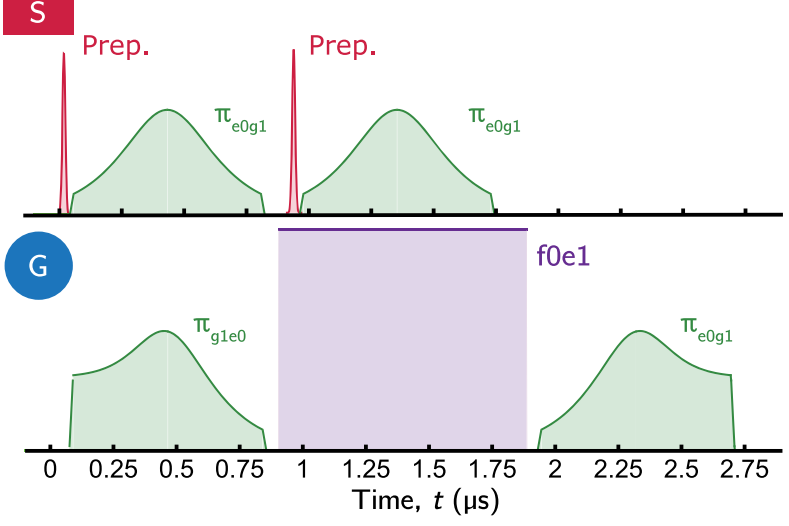

FIG. 13. Timing diagram of the pulses applied to the gate qubit $(G$, blue circle) and the source qubit ( $S$, red rectangle) for quantum process tomography of (a) single-photon gates and (b) the CPHASE gate. Depicted are the envelopes of the pulses on the charge line of the source (red) and the gate qubit (blue), as well as the envelopes of the flux pulses driving the $|e 0\rangle \leftrightarrow|g 1\rangle$ transition with the flux line of the respective coupler (green) and the $|f 0\rangle \leftrightarrow|e 1\rangle$ transition via the flux line of the coupler of the gate qubit (purple).

the gate qubit, such that the timing of the photonic qubits is independent of the applied gate. Since, in addition, the photonic qubits also have the same temporal shape after reemission from the gate qubit for all single-qubit gates, the encoding of the photonic qubits does not depend on the applied gate. The $T$ gate is realized as a virtual $T$ gate after waiting $t=36 \mathrm{~ns}$ by changing the reference frame for the subsequent coupler pulse $I_{\xi}(t)$, effectively adding a phase of $\pi / 8$ to $I_{\xi}(t)$.

During the controlled-phase gate, we apply a modulated square pulse to drive the $|f 0\rangle \leftrightarrow|e 1\rangle$ transition. We turn off this drive during idle times to avoid unwanted interactions between the photonic qubit and the gate qubit. The temporal shape of a photonic qubit reflected off of the gate qubit is then independent of the state of the gate qubit. To calibrate the controlled-phase gate, we sweep frequency and pulse duration of the modulated square pulse and read out the remaining populations of the gate qubit; see Fig. 14. Since the duration of the experiment is much shorter than (a)

(b)

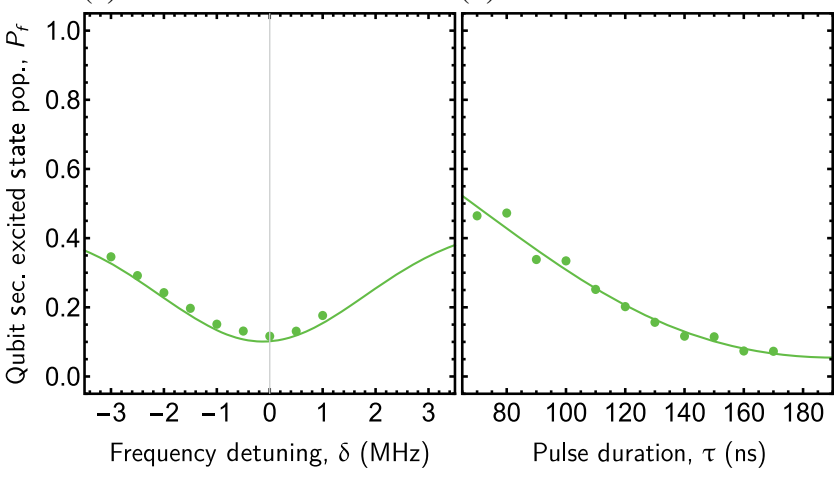

FIG. 14. (a),(b) Second excited state population $P_{f}$ after preparing the gate qubit in the second excited state and applying a modulated flux pulse with a square envelope and a fixed (normalized) amplitude of $A=1$. (a) Fixed pulse duration $\tau=170 \mathrm{~ns}$, plotted vs flux pulse frequency detuning $\delta$ from the $|f 0\rangle \leftrightarrow|e 1\rangle$ transition. The solid line is a Gaussian fit to the data. (b) On resonance $\delta=0$, plotted vs flux pulse duration $\tau$. The solid line is a fit to the data based on a driven two-level system decaying at a rate $\kappa_{G} / 2 \pi=2.1 \mathrm{MHz}$.

$T_{1}$, we consider a driven two-level Rabi-model in the $|e\rangle-$ $|f\rangle$ subspace to extract an interaction strength of $g / 2 \pi=$ 1.6 $\mathrm{MHz}$ from the measured populations in Fig. 14(b).

\section{APPENDIX D: MASTER EQUATION SIMULATIONS}

To simulate single-qubit process maps and calibration routines, we use a master equation approach, extending the work done in Ref. [38]. For all master equation simulations, we use the PYTHON package QuTiP [57].

To simplify the simulation, we model both the gate and the source qubit as anharmonic resonators with three energy levels and anharmonicity $\alpha$ coupled to a converter mode with two energy levels. In a frame rotating at both the $|g\rangle \leftrightarrow|e\rangle$ frequency $\omega_{g e} / 2 \pi$ of the qubit and the $|0\rangle \leftrightarrow|1\rangle$ frequency $\omega_{01} / 2 \pi$ of the respective converter mode, driving the $|e 0\rangle \leftrightarrow|g 1\rangle$ transition can be modeled by a resonant coupling term. We thus arrive at the Hamiltonian $H$ :

$$
\begin{aligned}
H= & \sum_{i=S, G}\left[\frac{\alpha_{i}}{2} a_{i}^{\dagger} a_{i}^{\dagger} a_{i} a_{i}\right] \\
& -\frac{i}{2} \kappa_{S} \kappa_{G} \eta_{\mathrm{loss}}\left(b_{S} b_{G}^{\dagger}-b_{G} b_{S}^{\dagger}\right) \\
& +J_{S}(t)\left(a_{S} b_{S}^{\dagger}+a_{S}^{\dagger} b_{S}\right) \\
& +J_{G}(t)\left(a_{G} b_{G}^{\dagger}+a_{G}^{\dagger} b_{G}\right),
\end{aligned}
$$

where the indices denote the source qubit $S$ and the gate qubit $G, a$ denotes the annihilation operator of the respective qubit, $b$ denotes annihilation operator of 
the converter mode, $\kappa$ denotes the coupling rates of the converter modes to the transmission line, and $\eta_{\text {loss }}=0.75$ is the photon transmission probability.

The first term (D1) in the Hamiltonian $H$ accounts for the detuned second excited state $|f\rangle$. We introduce the time-dependent coupling terms (D3) and (D4) to model the parametric coupling via the $|e 0\rangle \leftrightarrow|g 1\rangle$ transition. The second term (D2) of the Hamiltonian, together with the collapse operators $[38,58]$

$$
\begin{aligned}
& c_{1}=\sqrt{\kappa_{S} \eta_{\text {loss }}} b_{S}+\sqrt{\kappa_{G}} b_{G}, \\
& c_{2}=\sqrt{\kappa_{S}}\left(1-\eta_{\text {loss }}\right) b_{S},
\end{aligned}
$$

introduces a directional coupling between the two converter modes [59] and thus models a circulator with $1-\eta_{\text {loss }}=$ $25 \%$ loss. We take into account decoherence by including collapse operators for dephasing and relaxation in our simulation. For the simulation, we use the measured device parameters as specified in Table II. To accurately model the experiment, we evolve the time-dependent coupling terms $J(t)$ in the simulations according to Eq. (B2), taking into account the truncation and parameters for the coupler pulses $I_{\xi}(t)$ used in the experiment.

In this way, we extract both the simulated process maps $\chi_{\text {sim }}$ (shown in Fig. 4) and the simulated excited state populations for the calibration routines (shown in Fig. 12). We do not account for imperfections in the emission process from the gate qubit, since a photon Fock state |1) emitted from the gate qubit serves as a reference for the reconstruction of the measured density matrices [32,37].

To obtain an estimate of single-qubit gate fidelities within reach in the near future, we use the same master equation simulations but assume higher coupling rates $(J / 2 \pi=\kappa / 2 \pi=20 \mathrm{MHz})$, lossless transmission from the source to the gate chip $\left(\eta_{\text {loss }}=1\right)$, and coherence rates similar to state-of-the-art transmon qubits [44]: $T_{1}=$ $100 \mu \mathrm{s}$ and $T_{2}=100 \mu \mathrm{s}$. We then obtain prospective single-qubit fidelities around $99 \%$, limited by decoherence $(0.3 \%)$ and errors due to mode matching $(0.3 \%)$. For the controlled-phase gate, assuming that loss is not a dominant error contribution, similar process fidelities are within reach, considering that, in the limit $g / \Gamma \approx \kappa / \Gamma \gg 1$ and $\Gamma \gg 1 / T_{1}, 1 / T_{2}$, infidelities due to decoherence and mode distortions vanish. To obtain a more accurate estimate of future two-qubit fidelities, an extension of the master equation simulation to two-qubit gates is, however, needed. Furthermore, the expected increase in fidelity remains to be demonstrated in future experiments.

[1] P. Kok, W. J. Munro, K. Nemoto, T. C. Ralph, J. P. Dowling, and G.J. Milburn, Linear Optical Quantum Computing with Photonic Qubits, Rev. Mod. Phys. 79, 135 (2007).
[2] H. J. Kimble, The Quantum Internet, Nature (London) 453, 1023 (2008).

[3] Z.-L. Xiang, M. Zhang, L. Jiang, and P. Rabl, Intracity Quantum Communication via Thermal Microwave Networks, Phys. Rev. X 7, 011035 (2017).

[4] J. I. Cirac, A. K. Ekert, S. F. Huelga, and C. Macchiavello, Distributed Quantum Computation over Noisy Channels, Phys. Rev. A 59, 4249 (1999).

[5] H. Pichler and P. Zoller, Photonic Circuits with Time Delays and Quantum Feedback, Phys. Rev. Lett. 116, 093601 (2016).

[6] N. H. Nickerson, J.F. Fitzsimons, and S. C. Benjamin, Freely Scalable Quantum Technologies Using Cells of 5-to-50 Qubits with Very Lossy and Noisy Photonic Links, Phys. Rev. X 4, 041041 (2014).

[7] S. Slussarenko and G. J. Pryde, Photonic Quantum Information Processing: A Concise Review, Appl. Phys. Rev. 6, 041303 (2019).

[8] E. Knill, R. Laflamme, and G. J. Milburn, A Scheme for Efficient Quantum Computation with Linear Optics, Nature (London) 409, 46 (2001).

[9] J.-W. Pan, Z.-B. Chen, C.-Y. Lu, H. Weinfurter, A. Zeilinger, and M. Żukowski, Multiphoton Entanglement and Interferometry, Rev. Mod. Phys. 84, 777 (2012).

[10] N. C. Menicucci, P. van Loock, M. Gu, C. Weedbrook, T. C. Ralph, and M. A. Nielsen, Universal Quantum Computation with Continuous-Variable Cluster States, Phys. Rev. Lett. 97, 110501 (2006).

[11] J. M. Arrazola et al., Quantum Circuits with Many Photons on a Programmable Nanophotonic Chip, Nature (London) 591, 54 (2021).

[12] P. Walther, K. J. Resch, T. Rudolph, E. Schenck, H. Weinfurter, V. Vedral, M. Aspelmeyer, and A. Zeilinger, Experimental One-Way Quantum Computing, Nature (London) 434, 169 (2005).

[13] M. V. Larsen, X. Guo, C. R. Breum, J. S. NeergaardNielsen, and U. L. Andersen, Deterministic Generation of a Two-Dimensional Cluster State, Science 366, 369 (2019).

[14] W. Asavanant, Y. Shiozawa, S. Yokoyama, B. Charoensombutamon, H. Emura, R. N. Alexander, S. Takeda, J.-i. Yoshikawa, N. C. Menicucci, H. Yonezawa, and A. Furusawa, Generation of Time-Domain-Multiplexed Two-Dimensional Cluster State, Science 366, 373 (2019).

[15] C. K. Hong, Z. Y. Ou, and L. Mandel, Measurement of Subpicosecond Time Intervals between Two Photons by Interference, Phys. Rev. Lett. 59, 2044 (1987).

[16] H. Weinfurter, Experimental Bell-State Analysis, Europhys. Lett. 25, 559 (1994).

[17] H.-J. Briegel, W. Dür, J. I. Cirac, and P. Zoller, Quantum Repeaters: The Role of Imperfect Local Operations in Quantum Communication, Phys. Rev. Lett. 81, 5932 (1998).

[18] L. Jiang, J. M. Taylor, K. Nemoto, W. J. Munro, R. Van Meter, and M. D. Lukin, Quantum Repeater with Encoding, Phys. Rev. A 79, 032325 (2009).

[19] W. J. Munro, A. M. Stephens, S. J. Devitt, K. A. Harrison, and K. Nemoto, Quantum Communication without the Necessity of Quantum Memories, Nat. Photonics 6, 1749 (2012).

[20] K. Azuma, K. Tamaki, and H.-K. Lo, All Photonic Quantum Repeaters, Nat. Commun. 6, 6787 (2015). 
[21] J. L. O’Brien, G. J. Pryde, A. G. White, T. C. Ralph, and D. Branning, Demonstration of an All-Optical Quantum Controlled-Not Gate, Nature (London) 426, 264 (2003).

[22] Z. Zhao, A.-N. Zhang, Y.-A. Chen, H. Zhang, J.-F. Du, T. Yang, and J.-W. Pan, Experimental Demonstration of a Nondestructive Controlled-Not Quantum Gate for Two Independent Photon Qubits, Phys. Rev. Lett. 94, 030501 (2005).

[23] H.-H. Lu, J. M. Lukens, B. P. Williams, P. Imany, N. A. Peters, A. M. Weiner, and P. Lougovski, A Controlled-Not Gate for Frequency-Bin Qubits, npj Quantum Inf. 5, 24 (2019).

[24] H.-P. Lo, T. Ikuta, N. Matsuda, T. Honjo, W. J. Munro, and H. Takesue, Quantum Process Tomography of a ControlledPhase Gate for Time-Bin Qubits, Phys. Rev. Applied 13, 034013 (2020).

[25] J. Zeuner, A. N. Sharma, M. Tillmann, R. Heilmann, M. Grfe, A. Moqanaki, A. Szameit, and P. Walther, IntegratedOptics Heralded Controlled-Not Gate for PolarizationEncoded Qubits, npj Quantum Inf. 4, 13 (2018).

[26] J. Carolan, C. Harrold, C. Sparrow, E. Martn-Lpez, N. J. Russell, J. W. Silverstone, P. J. Shadbolt, N. Matsuda, M. Oguma, M. Itoh, G. D. Marshall, M. G. Thompson, J. C. F. Matthews, T. Hashimoto, J. L. OBrien, and A. Laing, Universal Linear Optics, Science 349, 711 (2015).

[27] J. Carolan, M. Mosheni, J. P. Olson, M. Prabhu, C. Chen, D. Bunandar, N. C. Harris, F. N. Wong, M. Hochberg, S. Lloyd et al., Variational Quantum Unsampling on a Quantum Photonic Processor, Nat. Phys. 16, 322 (2020).

[28] H.-S. Zhong et al., Quantum Computational Advantage Using Photons, Science 370, 1460 (2020).

[29] L.-M. Duan and H. J. Kimble, Scalable Photonic Quantum Computation through Cavity-Assisted Interactions, Phys. Rev. Lett. 92, 127902 (2004).

[30] B. Hacker, S. Welte, G. Rempe, and S. Ritter, A PhotonPhoton Quantum Gate Based on a Single Atom in an Optical Resonator, Nature (London) 536, 193 (2016).

[31] D. Tiarks, S. Schmidt-Eberle, T. Stolz, G. Rempe, and S. Drr, A Photon-Photon Quantum Gate Based on Rydberg Interactions, Nat. Phys. 15, 124 (2019).

[32] J.-C. Besse, K. Reuer, M. C. Collodo, A. Wulff, L. Wernli, A. Copetudo, D. Malz, P. Magnard, A. Akin, M. Gabureac, G. J. Norris, J. I. Cirac, A. Wallraff, and C. Eichler, Realizing a Deterministic Source of Multipartite-Entangled Photonic Qubits, Nat. Commun. 11, 4877 (2020).

[33] J. Ilves, S. Kono, Y. Sunada, S. Yamazaki, M. Kim, K. Koshino, and Y. Nakamura, On-Demand Generation and Characterization of a Microwave Time-Bin Qubit, npj Quantum Inf. 6, 34 (2020).

[34] P. Campagne-Ibarcq, E. Zalys-Geller, A. Narla, S. Shankar, P. Reinhold, L. Burkhart, C. Axline, W. Pfaff, L. Frunzio, R. J. Schoelkopf, and M. H. Devoret, Deterministic Remote Entanglement of Superconducting Circuits through Microwave Two-Photon Transitions, Phys. Rev. Lett. 120, 200501 (2018).

[35] J.-C. Besse, S. Gasparinetti, M. C. Collodo, T. Walter, P. Kurpiers, M. Pechal, C. Eichler, and A. Wallraff, SingleShot Quantum Non-demolition Detection of Individual Itinerant Microwave Photons, Phys. Rev. X 8, 021003 (2018).
[36] S. Kono, K. Koshino, Y. Tabuchi, A. Noguchi, and Y. Nakamura, Quantum Non-demolition Detection of an Itinerant Microwave Photon, Nat. Phys. 14, 546 (2018).

[37] C. Eichler, D. Bozyigit, and A. Wallraff, Characterizing Quantum Microwave Radiation and Its Entanglement with Superconducting Qubits Using Linear Detectors, Phys. Rev. A 86, 032106 (2012).

[38] P. Kurpiers, P. Magnard, T. Walter, B. Royer, M. Pechal, J. Heinsoo, Y. Salathé, A. Akin, S. Storz, J.-C. Besse, S. Gasparinetti, A. Blais, and A. Wallraff, Deterministic Quantum State Transfer and Remote Entanglement Using Microwave Photons, Nature (London) 558, 264 (2018).

[39] M. Pechal, L. Huthmacher, C. Eichler, S. Zeytinoğlu, A. A. Abdumalikov, Jr., S. Berger, A. Wallraff, and S. Filipp, Microwave-Controlled Generation of Shaped Single Photons in Circuit Quantum Electrodynamics, Phys. Rev. X 4, 041010 (2014).

[40] O. Morin, M. Körber, S. Langenfeld, and G. Rempe, Deterministic Shaping and Reshaping of Single-Photon Temporal Wave Functions, Phys. Rev. Lett. 123, 133602 (2019).

[41] S. Lloyd, Almost Any Quantum Logic Gate Is Universal, Phys. Rev. Lett. 75, 346 (1995).

[42] I. L. Chuang and M. A. Nielsen, Prescription for Experimental Determination of the Dynamics of a Quantum Black Box, J. Mod. Opt. 44, 2455 (1997).

[43] M. C. Collodo, J. Herrmann, N. Lacroix, C. K. Andersen, A. Remm, S. Lazar, J.-C. Besse, T. Walter, A. Wallraff, and C. Eichler, Implementation of Conditional Phase Gates Based on Tunable zz Interactions, Phys. Rev. Lett. 125, 240502 (2020).

[44] R. Barends et al., Superconducting Quantum Circuits at the Surface Code Threshold for Fault Tolerance, Nature (London) 508, 500 (2014).

[45] D. C. McKay, S. Filipp, A. Mezzacapo, E. Magesan, J. M. Chow, and J. M. Gambetta, Universal Gate for FixedFrequency Qubits via a Tunable Bus, Phys. Rev. Applied 6, 064007 (2016).

[46] F. Lecocq, L. Ranzani, G. A. Peterson, K. Cicak, R. W. Simmonds, J. D. Teufel, and J. Aumentado, Nonreciprocal Microwave Signal Processing with a Field-Programmable Josephson Amplifier, Phys. Rev. Applied 7, 024028 (2017).

[47] B. J. Chapman, E. I. Rosenthal, J. Kerckhoff, B. A. Moores, L. R. Vale, J. A. B. Mates, G. C. Hilton, K. Lalumière, A. Blais, and K. W. Lehnert, Widely Tunable On-Chip Microwave Circulator for Superconducting Quantum Circuits, Phys. Rev. X 7, 041043 (2017).

[48] P. Kurpiers, M. Pechal, B. Royer, P. Magnard, T. Walter, J. Heinsoo, Y. Salathé, A. Akin, S. Storz, J.-C. Besse, S. Gasparinetti, A. Blais, and A. Wallraff, Quantum Communication with Time-Bin Encoded Microwave Photons, Phys. Rev. Applied 12, 044067 (2019).

[49] H. Pichler, S. Choi, P. Zoller, and M. D. Lukin, Universal Photonic Quantum Computation via Time-Delayed Feedback, Proc. Natl. Acad. Sci. U.S.A. 114, 11362 (2017).

[50] K. Wan, S. Choi, I. H. Kim, N. Shutty, and P. Hayden, FaultTolerant Qubit from a Constant Number of Components, arXiv:2011.08213.

[51] S. Krinner, S. Storz, P. Kurpiers, P. Magnard, J. Heinsoo, R. Keller, J. Lütolf, C. Eichler, and A. Wallraff, Engineering 
Cryogenic Setups for 100-Qubit Scale Superconducting Circuit Systems, Eur. Phys. J. Quantum Technol. 6, 2 (2019).

[52] C. Eichler, Y. Salathe, J. Mlynek, S. Schmidt, and A. Wallraff, Quantum-Limited Amplification and Entanglement in Coupled Nonlinear Resonators, Phys. Rev. Lett. 113, 110502 (2014).

[53] J. Koch, T. M. Yu, J. Gambetta, A. A. Houck, D. I. Schuster, J. Majer, A. Blais, M. H. Devoret, S. M. Girvin, and R. J. Schoelkopf, Charge-Insensitive Qubit Design Derived from the Cooper Pair Box, Phys. Rev. A 76, 042319 (2007).

[54] B. R. Mollow, Power Spectrum of Light Scattered by TwoLevel Systems, Phys. Rev. 188, 1969 (1969).

[55] C. Lang, D. Bozyigit, C. Eichler, L. Steffen, J. M. Fink, A. A. Abdumalikov, Jr., M. Baur, S. Filipp, M. P. da Silva, A. Blais, and A. Wallraff, Observation of Resonant Photon Blockade at Microwave Frequencies Using Correlation
Function Measurements, Phys. Rev. Lett. 106, 243601 (2011).

[56] F. Motzoi, J. M. Gambetta, P. Rebentrost, and F. K. Wilhelm, Simple Pulses for Elimination of Leakage in Weakly Nonlinear Qubits, Phys. Rev. Lett. 103, 110501 (2009).

[57] J. R. Johansson, P. D. Nation, and F. Nori, QuTiP 2: A Python Framework for the Dynamics of Open Quantum Systems, Comput. Phys. Commun. 184, 1234 (2013).

[58] A. H. Kiilerich and K. Mølmer, Input-Output Theory with Quantum Pulses, Phys. Rev. Lett. 123, 123604 (2019).

[59] H. J. Carmichael, Quantum Trajectory Theory for Cascaded Open Systems, Phys. Rev. Lett. 70, 2273 (1993).

Correction: A grant number was missing in the Acknowledgments section has been inserted. 